\title{
A case-controlled study of the sexual health needs of lesbians
}

\author{
C J Skinner, J Stokes, Y Kirlew, J Kavanagh, G E Forster
}

Objectives: To evaluate and compare the range of genital infections diagnosed in a group of lesbians attending an inner city genitourinary clinic with a control group of heterosexual women attending the same clinic.

Setting: The Ambrose King Centre, the Royal London Hospital, a genitourinary clinic within which the Audre Lorde Clinic, a specialist sexual health clinic for women identifying as lesbians, is operated.

Subjects: Two hundred and forty one women attending the specialist clinic between October 1993 and September 1994. Heterosexual controls matched for age and ethnicity were selected from the same time period.

Methods: A retrospective case note analysis was made of 241 lesbians and 241 matched heterosexual controls. Data were collected on age, ethnicity, symptoms, diagnoses and services used.

Results: An infection was diagnosed in $129(65 \%)$ of the lesbians and $126(62 \%)$ of the heterosexual women. Only $23(10 \%)$ of the lesbians exclusively practised same gender sexual contact. Genital herpes $(p=0.05)$ and genital warts $(p=0.005)$ were more common in the heterosexual women. Gonorrhoea and chlamydia infection were infrequent diagnoses in both groups, occurring in four $(2 \%)$ lesbians and $14(7 \%)$ heterosexuals $(p=0.05)$. Bacterial vaginosis occurred in $65(33 \%)$ of the lesbians and $27(13 \%)$ of the heterosexuals $(p<0.0001)$. Cervical cytology abnormalities were uncommon but only found in the lesbians.

Conclusions: Screening for genital infections in lesbians is appropriate. The high prevalence of bacterial vaginosis in this group is unexplained but suggests a possible sexual transmission. Lesbians should be included within the cervical cytology screening programme.

(Genitourin Med 1996;72:277-280)

Keywords: lesbian; sexually transmitted diseases; bacterial vaginosis; cervical cytology

\section{Introduction}

Research into sexually transmitted diseases (STDs) and HIV infection amongst homosexuals has almost exclusively concentrated on homosexual men. The lack of research on lesbians probably reflects a number of factors including difficulty in disclosing sexual orientation in a medical setting, the lower numbers of women identifying as homosexual in comparison with men and the risk of infection relating most closely to penile penetration of the vagina or rectum. The studies conducted in lesbians have been weakened by small numbers, selection bias and methodological limitations. A study of 148 lesbians from San Francisco found no cases of syphilis, gonorrhoea, chlamydia or herpes infection but two presumptive cases of pelvic inflammatory disease. ${ }^{1}$ Two American questionnaire based studies have found similarly low rates of infection. ${ }^{2}{ }^{3}$ Edwards reported on the findings in an unselected sample of 27 lesbians attending a London clinic and found a relatively high rate of viral infections (10), and bacterial vaginosis in 13 patients. ${ }^{4}$ There are a few case reports of HIV infection possibly resulting from femaleto-female sexual contact. ${ }^{5-7}$ However, assuming that lesbians are a low risk group for the acquisition of STDs takes no account of other risk behaviours including injecting drug use and unprotected heterosexual coitus. This was demonstrated by a questionnaire based study of lesbians in Turin which found $6 \%$ of the sample to be infected with HIV-1 and a rela- tively high rate of STDs. Intravenous drug use was the most likely route of HIV acquisition in this study. ${ }^{8}$

With this inherent knowledge of their own sexual behaviours, lesbians perceived a need for sexual health advice and screening. This has led to the setting up of four specialist sexual health clinics for lesbians over the last three and a half years in the United Kingdom. The Audre Lorde Clinic, at the Royal London Hospital, is one such clinic. We set out to examine the prevalence of STDs and other infections amongst lesbians attending this clinic in comparison with heterosexual women attending the main genitourinary clinic in order to evaluate the need for STD screening in this population.

\section{Subjects and methods}

The case notes of all lesbians who attended the Audre Lorde Clinic during its first year (October 1993-September 1994) were reviewed retrospectively. The clinic operates an open access, booked appointments system. Data were collected using a standardised proforma on age, ethnicity, sexual history, reasons for presentation, symptoms, investigations, diagnoses made, the use of other services within the clinic and outward referral to other medical specialities. A control population of self-identified heterosexual women attending the main genitourinary clinic as self-referred, new patients was selected from the same time 
Table 1 Sexual behaviour characteristics

\begin{tabular}{lccc}
\hline & & \multicolumn{2}{l}{ Heterosexual contact } \\
\cline { 3 - 4 } Time & Same gender sexual contact & $\begin{array}{l}\text { Lesbians* } \\
N=218(\%)\end{array}$ & $\begin{array}{l}\text { Heterosexuals } \\
\text { Lesbians } N=227(\%)\end{array}$ \\
\hline $1-7$ days & $148(65 \%)$ & $0(0 \%)$ & $135(58 \%)$ \\
$8-30$ days & $42(19 \%)$ & $0(0 \%)$ & $63(27 \%)$ \\
$31-90$ days & $14(6 \%)$ & $4(2 \%)$ & $17(7 \%)$ \\
91 days-1 year & $13(6 \%)$ & $9(4 \%)$ & $9(4 \%)$ \\
$>1$ year & $10(4 \%)$ & $169(77 \%)$ & $8(4 \%)$ \\
\hline
\end{tabular}

$\star 36(17 \%)$ lesbians not known when last heterosexual contact occurred.

period. Patients were matched for age and ethnicity using the clinic registration book. The same standardised proforma was used for data collection.

A standardised STD screen was offered to all patients: cervical and urethral Gramstained smear and culture for Neisseria gonorrhoeae, cervical swab for Chlamydia trachomatis by ELISA and high vaginal samples for Trichomonas vaginalis by wet preparation. Gram-stained vaginal preparations were taken for Candida sp identification and subsequent culture. Bacterial vaginosis was diagnosed on the basis of Amsel's criteria. ${ }^{9}$ Cervical cytology was performed with an Aylesbury spatula and/or cytobrush, as appropriate. Patients were screened for treponemal disease using a combination of the non-specific Venereal Diseases Research Laboratory test and the specific Treponema pallidum haemagglutination assay. Other tests, including herpes simplex cultures, were performed as clinically indicated.

Data were processed with SPSS (Statistical Package for Social Sciences) software, version $5 \cdot 0$ (SPSS Inc, Chicago, Illinois, USA).

\section{Results}

From October 1993 to September 1994, 241 lesbians and 241 matched heterosexual controls were reviewed.

\section{Group characteristics and sexual behaviour}

The mean age in years was 32 (range 18-53) for the lesbians and 31 (range 17-57) for the controls. The two populations were identically matched for ethnicity with the majority 221 (92\%) identified as Caucasians and $12(5 \%)$ identified as Black. Of the 241 women selfidentifying as lesbians, 227 (94\%) gave a clear history of same gender sexual contact, for the remaining $14(6 \%)$ no history was docu-

Table 2 Genital infections and other diagnoses in lesbians and heterosexual women

\begin{tabular}{|c|c|c|c|c|c|}
\hline Diagnosis/condition & $\begin{array}{l}\text { Lesbians } \\
\text { (\%) }\end{array}$ & $N=199$ & $\begin{array}{l}\text { Heterosexuals } \\
(\%)\end{array}$ & $N=204$ & $p$ value \\
\hline Bacterial vaginosis & 65 & $33 \%$ & 27 & $13 \%$ & $<0.0001$ \\
\hline Calbicans & 44 & $22 \%$ & 43 & $21 \%$ & \\
\hline $\begin{array}{l}C \text { trachomatis } \\
\text { Genital warts }\end{array}$ & 3 & $2 \%$ & 10 & $5 \%$ & \\
\hline $1^{\text {st }}$ Episode & 1 & $0.5 \%$ & 14 & $7 \%$ & \multirow{2}{*}{0.0005} \\
\hline Recurrent & 0 & 0 & 3 & $1 \%$ & \\
\hline \multirow{2}{*}{\multicolumn{6}{|c|}{ Herpes simplex }} \\
\hline & & & & & \\
\hline Primary & 2 & $1 \%$ & 10 & $5 \%$ & \multirow[t]{2}{*}{0.05} \\
\hline Recurrent & 2 & $1 \%$ & 4 & $2 \%$ & \\
\hline$M$ contagiosum & 4 & $2 \%$ & 0 & 0 & ns \\
\hline$T$ vaginalis & 4 & $2 \%$ & 4 & $2 \%$ & ns \\
\hline
\end{tabular}

mented in the notes. Within the last month, $190(78 \%)$ of the lesbians had experienced same gender sexual contact. Of the lesbians, $218(91 \%)$ had experienced heterosexual intercourse but in only four $(2 \%)$ had this occurred in the last 90 days and none within the last 60 days. In 34 (14\%) of the lesbians, heterosexual intercourse had not occurred within the last 10 years. All of the heterosexual women were sexually active, although the time of last heterosexual intercourse was not recorded in nine $(4 \%)$ women. One hundred and ninety eight $(82 \%)$ had experienced vaginal intercourse in the last 30 days. None of the women in the heterosexual group gave a history of same gender sexual contact (table 1).

\section{Reasons for presentation}

The majority of women in each group were symptomatic at presentation: $131(54 \%)$ of the lesbian group and $149(62 \%)$ of the heterosexual group. Additional reasons for presentation reflected the different emphases of the two clinics in that lesbians were more likely to request cervical cytology $91(38 \%)$ and breast examination $44(18 \%)$, whereas only nine $(4 \%)$ heterosexual women specifically requested cervical cytology and none of the heterosexual women requested breast examination. Similarly $52(22 \%)$ heterosexuals presented with contact slips compared with six $(3 \%)$ lesbians, and seven $(3 \%)$ heterosexual women asked for emergency contraception. HIV testing was specifically requested by $10 \%$ of both groups and performed in 49 $(20 \%)$ of the lesbians and $33(14 \%)$ of the heterosexual women, $(p=0.05)$.

STD diagnoses and cytology results

The majority of women agreed to have a full STD screen: $199(83 \%)$ of the lesbians and $204(85 \%)$ of the heterosexuals. Of those examined, $129(65 \%)$ of the lesbians and 126 $(62 \%)$ of the heterosexual women had an infection diagnosed. In addition, $52(26 \%)$ of the heterosexual women received epidemiological treatment as contacts of men with nonspecific urethritis (table 2$)$. Sixty five $(33 \%)$ of the lesbians and $27(13 \%)$ of the heterosexual women had a diagnosis of bacterial vaginosis, $(\mathrm{p}<0.001)$. Candida $s p$ were diagnosed in similar proportions between the groups. Both genital herpes and genital warts were significantly more common in the heterosexual women. The majority of the cases were first episode and all the lesbians except one with primary herpes simplex had been heterosexually active. The one lesbian with genital warts had not experienced heterosexual intercourse for eight years. However, the other sexually transmitted diseases, gonorrhoea, chlamydia and trichomoniasis were diagnosed infrequently in both groups; the higher rates in the heterosexual women did not reach statistical significance. None of the lesbians in whom these diagnoses were made had experienced heterosexual intercourse in the preceding three years. Of the 23 women who reported exclusive same gender sexual contact, 13 were examined and bacterial vaginosis was diagnosed in seven, 
Candida sp in two, herpes simplex in one and $T$ vaginalis in one.

Significantly more of the lesbians [149 $(62 \%)$ ] underwent cytology compared with heterosexual women [96 (40\%)], $\mathrm{p}=0.0001$. The only abnormal cervical smears were found in the lesbians; one showed borderline change, one moderate dyskaryosis and two severe dyskaryosis. All of these women had experienced heterosexual intercourse. The two women with severe dyskaryosis had not had sexual contact with men for 16 and 20 years respectively. The woman with moderate dyskaryosis also had cytological evidence of human papilloma virus. She had no evidence of genital warts although her current female sexual partner was receiving treatment for genital warts. Her last male sexual contact had been 20 months previously. The woman with borderline change reported her last male contact four years previously.

\section{Utilisation of other services}

Of the lesbians, $36(15 \%)$ compared with six $(2 \%)$ of the heterosexual women were referred to other medical specialities, $p=0.001$. The majority of the referrals were to gynaecology, [12 (5\%)] and to breast screening services [16 $(7 \%)$ ]. A further $39(16 \%)$ of the lesbians were seen by the clinic health advisors, mainly for pre-test counselling for HIV infection. This compared with $26(11 \%)$ of the heterosexual women seeing the health advisors.

\section{Discussion}

This study shows that in absolute terms there was no difference in the number of genital infections diagnosed in both lesbian and heterosexual women, supporting the need for genitourinary services for lesbians. However, a perception still exists that lesbians present with "well women" concerns predominately requesting cervical cytology and breast screening. Our data do not wholly support this view with the majority of lesbians (54\%) presenting with symptoms. Specific requests for cervical cytology and breast examination were nevertheless much commoner among the lesbians and probably represent failure to utilise primary medical care. The demographic characteristics of the lesbians were not typical of the females attending the main genitourinary clinic where the average age is lower and a greater ethnic diversity reflects the local population. These findings mirror the experiences of other investigators. ${ }^{14}$ It was for this reason that we matched the control group for age and ethnicity.

There were significant differences in the infections diagnosed between the two groups with an high rate of bacterial vaginosis amongst the lesbians. This has been found at another clinic for lesbians. ${ }^{10}$ The aetiology of bacterial vaginosis is poorly understood and felt to be multifactorial. We are unable to draw any firm conclusions as our data were retrospective and information on sexual practice, the use of lubricants and sex toys was not routinely collected. The possibility of sexual transmission through exchange of vaginal secretions or oro-genital contact is suggested and warrants further study.

Other authors have suggested that femaleto-female transmission of $N$ gonorrhoeae and $C$ trachomatis are unlikely in view of their failure to demonstrate either organism in lesbians ${ }^{1-3}$ and because of the known routes of transmission of these infections. We demonstrated Gram negative intracellular diplococci in cervical material from one woman who had not had heterosexual contact for more than three years. The microbiology laboratory confirmed this as highly suggestive of $N$ gonorrhoeae but the culture was negative. We also found three positive ELISAs for chlamydia where heterosexual contact had been a minimum of nine years previously. Confirmatory tests were not performed. We examined one of the female contacts of an index case of chlamydial infection and the partner was negative, but femaleto-female transmission is a possible explanation. However, both infections are known to have long periods of potential asymptomatic carriage. ${ }^{1112}$ The diagnosis of trichomoniasis was made in four women in each group and two of the lesbians were sexual partners. The transmission of $T$ vaginalis is considered possible via fomites or inoculation with infected vaginal secretions. Our findings are therefore consistent with other authors who have shown female-to-female transmission of $T$ vaginalis. ${ }^{313}$ The small number of cases of genital warts and herpes in the lesbians makes any firm conclusions on femaleto-female transmission difficult, although one case of herpes was found in a woman with exclusive female sexual contact.

The cervical cytology findings are difficult to interpret in view of the small number of abnormal results and the incomplete sexual and previous cytology histories of the women studied. The higher demand for cervical cytology amongst the lesbians probably reflects a failure to utilise other medical services. However, genital warts can be transmitted through non-penetrative intercourse. We would suggest that lesbians should not be excluded from the cervical screening programme especially if they have been bisexually active.

The risks of female-to-female transmission of HIV infection are unknown although extrapolation from heterosexual and male homosexual sexual activity plus the paucity of case reports suggests it to be low. Indeed a small study of $18 \mathrm{HIV}$ seropositive lesbians failed to demonstrate any seroconversion in their regular sexual partners over a limited six month follow up period. ${ }^{14}$ In our study, 49 (20\%) lesbians had an HIV test compared with $33(14 \%)$ heterosexual women, $(p=$ $0 \cdot 05)$. All test results were negative. This higher rate of testing amongst lesbians suggests a perception of risk or at least a greater awareness of safer sex issues. Further prospective work is needed to elucidate this.

In summary, this study demonstrates comparable rates of infection between lesbian and heterosexual women with a diversity of diag- 
noses being made. We recommend offering full STD screens to lesbians, in particular to those with a past history of heterosexual intercourse. The higher rate of bacterial vaginosis amongst lesbians is as yet unexplained but may provide an opportunity for further study of the aetiology of this condition.

1 Robertson P, Schachter J. Failure to identify venereal disease in a lesbian population. Sex Transm Dis 1981;8: 75-6.

2 Johnson SR, Smith EM, Guenther SM. Comparison of gynaecological health care problems between lesbians
and bisexual women. A survey of 2,345 women. $\mathcal{f}$ Reprod and bisexual women.

3 Degen K, Waitkevicz HJ. Lesbian health issues. Br $\mathcal{F}$ Sex Med 1982;May:40-7.

4 Edwards A, Thin RN. Sexually transmitted diseases in lesbians. Int F STD AIDS 1990;1:178-81.

5 Monzon OT, Capellan JMB. Female-to-female transmission of HIV (letter). Lancet 1987;2:40-1.
6 Marmor M, Weiss LR, Lyden M, et al. Possible female-tofemale transmission of Human Immunodeficiency Virus. Ann Intern Med 1986;105:969.

7 Chu SY, Conti L, Schable BA, et al. Female-to-female sexual contact and HIV transmission. $\mathcal{F} A M A$ 1994;272:433.

8 Raiteri R, Fora R, Gioannini P, et al. Seroprevalence, risk factors and attitude to HIV-1 in a representative sample of lesbians in Turin. Genitourin Med 1994;70:200-5.

9 Amsel R, Totten PA, Spiegal CA, et al. Nonspecific vaginitis: diagnostic criteria and microbiol and epidemiological associations. Am $\mathcal{F}$ Med 1983;74:4-22.

10 Varney $\mathrm{P}$, McCaffrey $M$, Taylor-Robinson $\mathrm{D}$, et al. Abnormal vaginal bacterial colonisation in lesbian women. MSSVD Spring Meeting 1994, abstract no. 91 .

11 McCormack WM, Alpert S, McComb DE, et al. Fifteen month follow-up study of women infected with month follow-up study of women infected with

12 Dunlop EMC, Darougar S, Treharne JD. Epidemiology of infection by serotypes $\mathrm{D}$ to $\mathrm{K}$ Chlamydia trachomatis. $\mathrm{Br} \mathfrak{f}$ Venereal Dis 1980;56:163-5.

13 Sivakumar K, De Silva AH, Basu Roy R. Trichomonas vaginalis infection in a lesbian. Genitourin Med 1989;65: 399-400.

14 Raiteri R, Fora R, Sinicco A. No HIV-1 transmission through lesbian sex (letter). Lancet 1994;2:270. 